\title{
The Frequency of Occult Intertrochanteric Fractures among Individuals with Isolated Greater Trochanteric Fractures
}

\author{
Jongho Noh, MD, Kee Haeng Lee, MD, Sehoon Jung, MD, Sunwook Hwang, MD \\ Department of Orthopedic Surgery, The Catholic University of Korea, Bucheon St. Mary's Hospital, Bucheon, Korea
}

Purpose: Isolated greater trochanteric (GT) fractures are often identified using plain radiography of patients with post-traumatic hip pain. In many cases, the fracture extends to form an occult intertrochanteric fracture. We conducted a study to determine the frequency of occult intertrochanteric fractures in patients diagnosed with isolated GT fractures using plain radiographs.

Materials and Methods: Among 3,017 individuals who visited our emergency department with a trauma-induced pertrochanteric femur fracture between July 2004 and March 2018, 100 patients diagnosed with isolated GT fractures using plain radiographs were retrospectively analyzed. Patients were divided into two groups, those with: i) isolated GT fractures (group A) and ii) occult intertrochanteric fractures (group B). In addition, plain radiographs, magnetic resonance imaging results, and treatment methods were further analyzed in each group. If surgery treatment was needed, it was performed by one surgeon, and in all cases, a 2-hole dynamic hip screw was used.

Results: Among the 100 cases of isolated GT fractures diagnosed using plain radiograph, additional examinations revealed that $10(10.0 \%)$ were suffering from isolated GT fractures alone, while the remaining 90 (90.0\%) were further diagnosed with occult intertrochanteric fracture. Gender, age, mechanism of injury, and bone mineral density did not correlate with fracture type.

Conclusion: In our analysis, $90 \%$ of injuries initially diagnosed as isolated GT fractures were found to extend into occult intertrochanteric fractures upon further examination with additional imaging modalities. Therefore, additional evaluation should be performed to test for the potential presence of occult intertrochanteric fractures and to establish appropriate treatment plans.

Key Words: Trochanteric fractures, Hip fractures, Closed fractures

Submitted: November 9, 2018 1st revision: December 17, 2018

Final acceptance: December 20, 2018

Address reprint request to

Kee Haeng Lee, MD

(https://orcid.org/0000-0002-2137-2992)

Department of Orthopedic Surgery, The Catholic University of

Korea, Bucheon St. Mary's Hospital, 327 Sosa-ro, Wonmi-gu,

Bucheon 14647, Korea

TEL: +82-32-340-2260 FAX: +82-32-340-2671

E-mail: keeleehiplagmail.com

This is an Open Access article distributed under the terms of the Creative Commons Attribution Non-Commercial License (http://creativecommons. org/licenses/by-nc/4.0) which permits unrestricted non-commercial use, distribution, and reproduction in any medium, provided the original work is properly cited.

\section{INTRODUCTION}

After trauma, isolated greater trochanteric fractures may occur in the femur. These fractures can be caused by an avulsion injury or by a direct blow-a mechanism usually seen in older adults ${ }^{1}$. When an isolated greater trochanteric fracture occurs, pain is usually local (involving only the hip), mild, and sometimes asymptomatic. However, if the pain is more severe than the pain associated with a typical isolated greater trochanteric fracture, especially if the pain increases during internal rotation, additional tests should be 


\section{Hip \& Pelvis}

Hip Pelvis 31(1): 23-32, 2019

conducted to rule out the presence of an occult intertrochanteric (ITC) fracture. There is no reported difference in the rate of long-term mortality when isolated trochanteric fractures are treated without the diagnosis of an occult fracture ${ }^{2)}$. However, occult ITC fractures increase the likelihood of fracture displacement, thereby increasing the proportion of surgical treatments and significantly decreasing the patient's activity ${ }^{3}$. Therefore, it is important to accurately identify the pattern of a fracture at a hospital visit for hip pain after a trauma and to plan and execute the most appropriate treatment.

It is well known that isolated greater trochanteric fractures are related to occult ITC fractures; many studies have been published relating to the diagnosis and treatment of these conditions ${ }^{4}$. However, little research has been conducted relating to the frequency of isolated greater trochanteric fractures diagnosed using plain radiographs extending to occult ITC fractures. Additionally, each of the few studies that do exist was conducted with small sample sizes.

The primary goal of our study is characterize the frequency of occult ITC in patients with preexisting radiographic evidence of isolated greater trochanteric fracture. This study includes a relatively large sample size (i.e., $\mathrm{n}=100$ ) and additional imaging assessments (e.g., magnetic resonance imagery [MRI]). The secondary goal is to identify any potential risk factors the occurrence of occult ITC.

\section{MATERIALS AND METHODS}

Between July 2004 and March 2018, 3,017 individuals visited our emergency department with a trauma-induced femur fracture. Among them, 100 patients (39 male, 61 female) were diagnosed, using plain radiographs, with isolated greater trochanteric fractures without pathologic or periprosthetic causes. These 100 cases were retrospectively analyzed for this study (Fig. 1).

The average age of all patients was 72.8 years $(66.02$ years for males, 77.2 years for females) with a range of 34 to 94 years (36-87 years for males, 34-94 years for females). Fractures occurred after a fall of fewer than 2 $m(n=90)$, a fall of greater than $2 \mathrm{~m}(n=6)$. and a traffic accident $(n=4)$. There were 47 cases involving the right femur and 53 cases involving the left femur.

For all patients, at least one additional assessment with an alternate imaging modality (e.g., MRI, computed tomography [CT], bone scan) was performed. The most common alternate procedure was MRI; however, patients with ITC fracture clearly visible on CT in the emergency department did not receive MRI. All images, including X-rays, CT scans, MRI scans, and

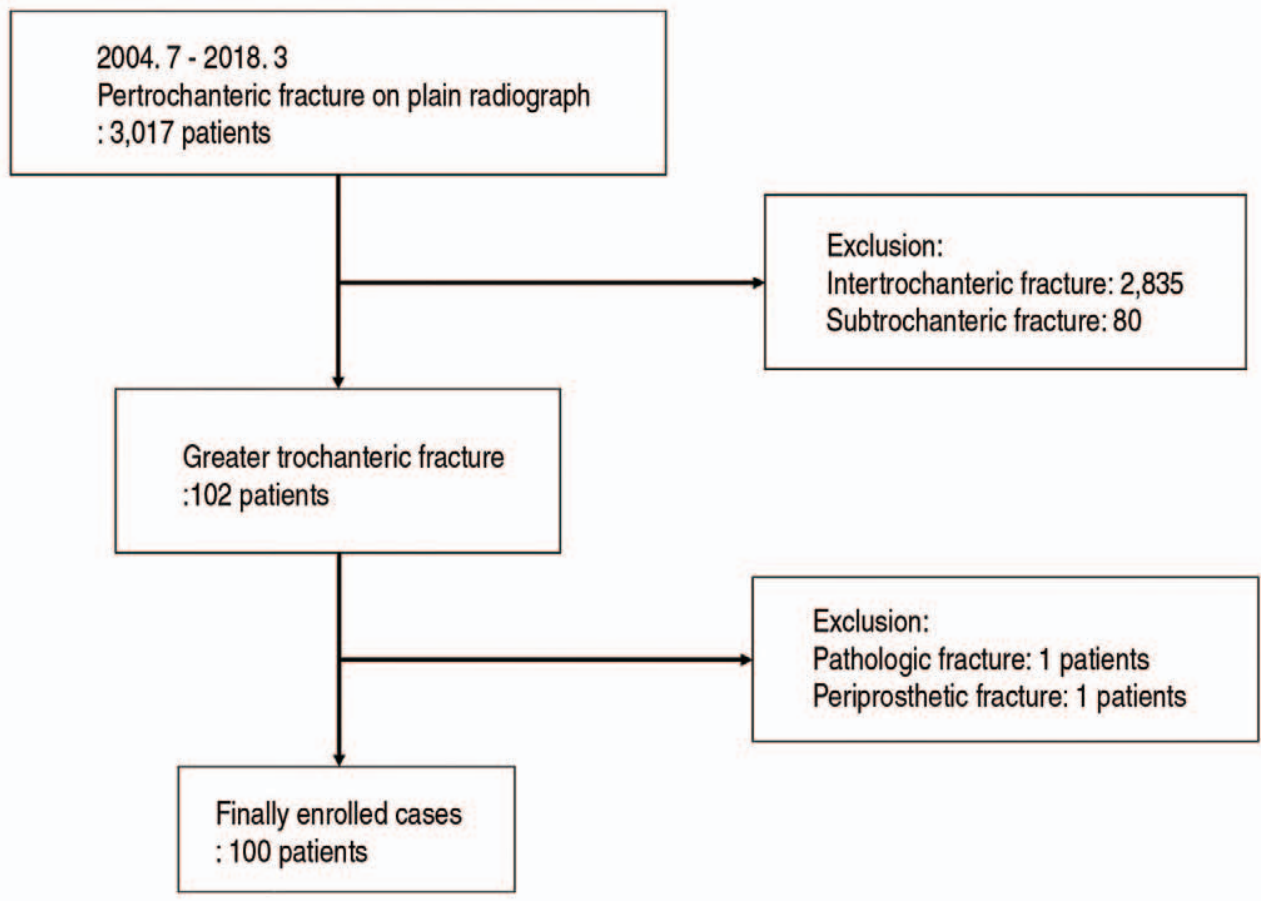

Fig. 1. Flow chart demonstrating how cases were selected and analyzed. 


\section{Hip \& Pelvis}

Jongho Noh et al. Isolated Greater Trochanteric Fracture with Occult Intertrochanteric Fracture

bone scans, were evaluated by two orthopedic surgeons and one radiologist.

MRI scans were performed in 92 cases (of these, 39 also received bone scans and seven also received $\mathrm{CT}$ scans), eight patients received CT scans alone. The average time interval from accident to examination was
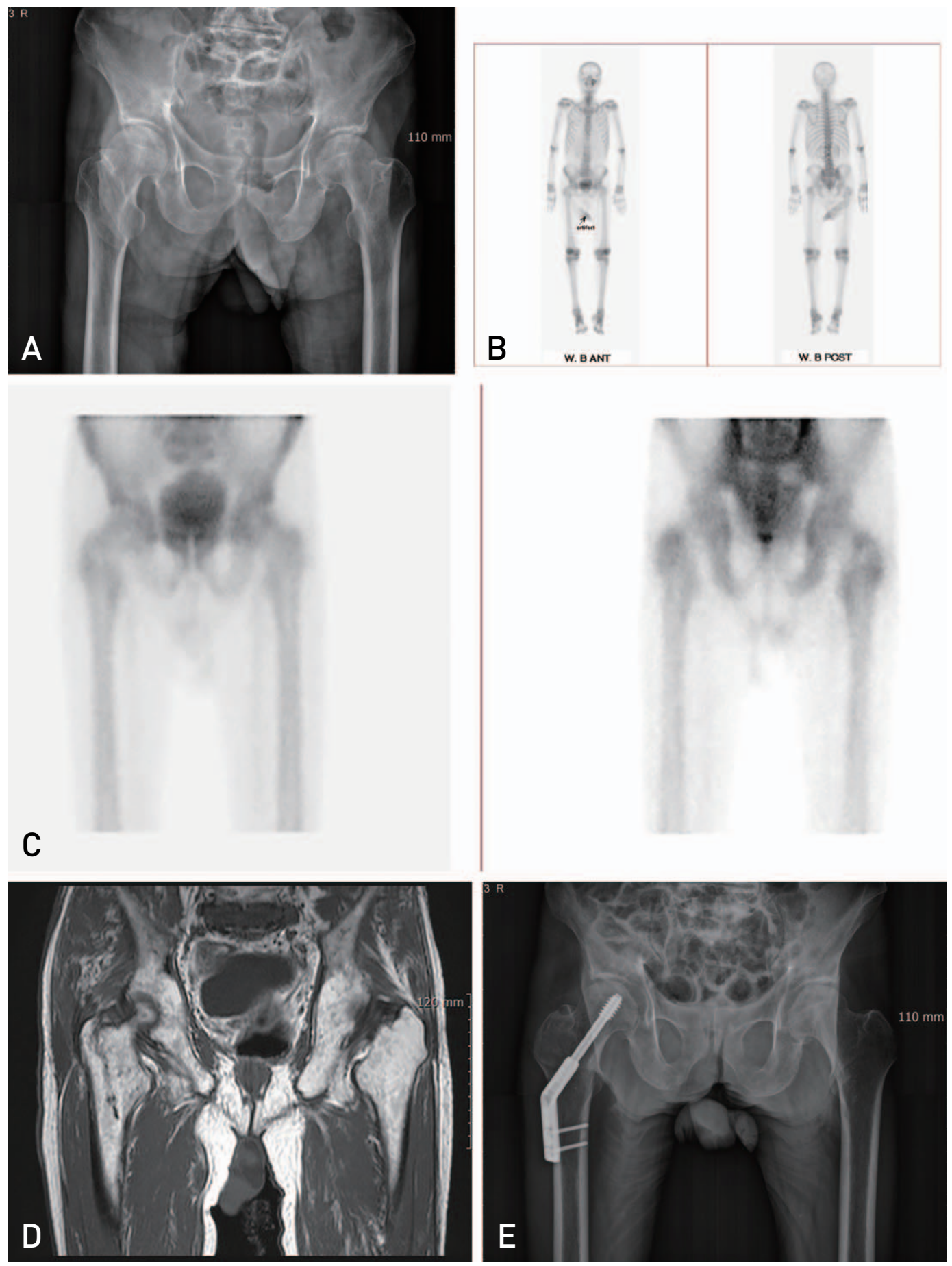

Fig. 2. An 87-year-old woman visited the hospital with right hip pain caused by a fall from height. (A) Plain radiography indicated an isolated greater trochanteric fracture. (B, C) A bone scan also revealed an isolated greater trochanteric fracture. (D) However, magnetic resonance imaging scans indicated that the fracture was more than half of the intertrochanteric area. (E) Surgical treatment was performed using two-hole dynamic hip screws.

W.B: whole body bone scan, ANT: anterior, POST: posterior. 


\section{Hip \& Pelvis}

Hip Pelvis 31(1): 23-32, 2019

0.47 days (range, 0-2 days) for CT scan, 2.65 days (range, 0-38 days) for MRI, and 5.5 days for bone scan (range, 1-9 days).

In the 39 cases wherein both MRI and bone scans were performed, 35 (89.7\%) revealed identical results, while 4 revealed conflicting results. In all 4 of the cases with conflicting results, the bone scan showed an isolated greater trochanteric fracture, whereas MRI showed an occult ITC fracture (Fig. 2).

$\mathrm{CT}$ and MRI were performed in 7 cases, and CT revealed the presence of an isolated greater trochanteric fracture in 3 cases. However, MRI showed occult ITC fractures more than halfway into the medial cortex in all 7 cases, and surgical treatment was performed in 4 cases.

An occult ITC fracture was defined as occurring when: i) the fracture line was extended to the ITC region on MRI, especially on T2-weighted images, ii) cortical breakage was observed in the ITC, iii) trabecular impaction was observed in the cancellous bone on CT, or iv) a hot uptake was observed in the ITC area on bone scan. Depending on the test results, these patients were divided into two groups, patients with an isolated greater trochanteric fracture (group A) and those with an occult ITC fracture (group B). In each group, sex, age, accident mechanism, direction, and bone mineral density (BMD), plain radiographs, MRI results, and treatment methods were further analyzed.

All patients in group A underwent conservative treatment. The conservative treatment entailed gradual introduction of weight bearing with a walker, without traction, after a period of bed rest for one to three weeks. Patients in group B who were in an operable state and who's MRIs and CT scans revealed occult ITC fractures that exceeded half the diameter of the metadiaphyseal region were operated upon. The operation was performed by one surgeon, and in all cases, a two-hole dynamic hip screw was used without reduction. The day after the operation, patients began rehabilitation with continuous passive motion, quadriceps strengthening exercise, tilting table, and P-bar. Within a week of the operation, patients began walker ambulation gradually. The patients visited clinics after one, three, and six months, and 1 year after surgery.

Statistical analyses were performed using the paired $t$ test, chi-square analysis, and Fisher's exact test. Statistical processing of all data was performed using IBM SPSS ver. 20 statistical software (IBM Corp., Armonk, NY, USA), and data were considered statistically significant if the $P$-value was less than 0.05 .

This article was approved by The Catholic University of Korea Catholic Medical Center at July 2, 2018 (project number, HC18RESI0049). That center was approved full accreditation by AAHRPP (Association for the Accreditation of Human Research Protection Program) at September 10, 2013.

\section{RESULTS}

There were 100 patients initially diagnosed with an isolated greater trochanteric fracture using plain radiograph. On plain radiography fractures were diagnosed as transverse fractures $(n=46)$, oblique fractures $(n=40)$, and comminuted fractures $(n=14)$. After further studies were conducted, patients were further
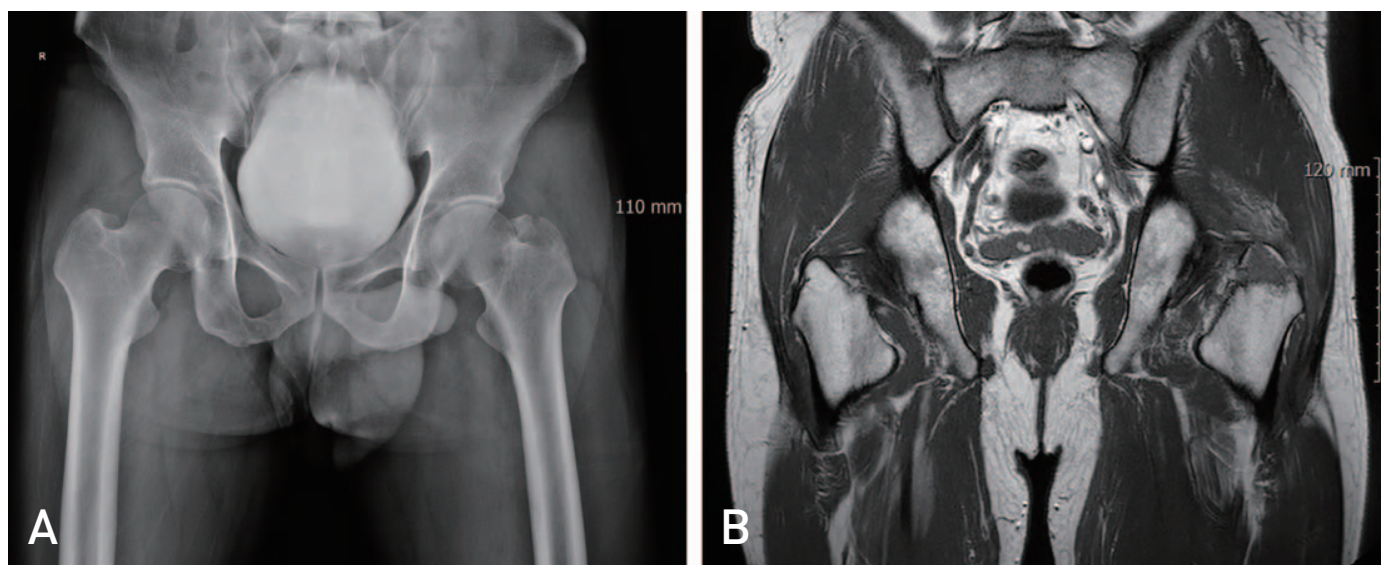

Fig. 3. A 36-year-old male patient visited the hospital with left hip pain following a traffic accident. Plain radiography (A) and magnetic resonance imaging $(B)$ each indicated an isolated greater trochanteric fracture, and conservative treatment was performed. 


\section{Hip \& Pelvis}

Jongho Noh et al. Isolated Greater Trochanteric Fracture with Occult Intertrochanteric Fracture

analyzed for the potential presence of isolated greater trochanteric fractures and/or occult ITC fractures.

MRI scans were performed in 92 cases (of these, 39 also received bone scans and seven also received $\mathrm{CT}$ scans), eight patients received CT scans alone. The average time interval from accident to examination was 0.47 days (range, 0-2 days) for CT scan, 2.65 days (range, 0-38 days) for MRI, and 5.5 days for bone scan (range, 1-9 days).

In the 39 cases wherein both MRI and bone scans were performed, 35 (89.7\%) revealed identical results, while 4 revealed conflicting results. In all 4 of the cases with conflicting results, the bone scan showed an isolated greater trochanteric fracture, whereas MRI showed an occult ITC fracture (Fig. 2).

$\mathrm{CT}$ and MRI were performed in 7 cases, and CT revealed the presence of an isolated greater trochanteric fracture in 3 cases. However, MRI showed occult ITC fractures more than halfway into the medial cortex in all 7 cases, and surgical treatment was performed in 4 cases.

Following further analysis, and as shown in Fig. 3, patients were divided into group A (isolated greater trochanteric fractures, $n=10$ ) or group $B$ (occult ITC fractures, $n=90$ ). As shown in Fig. 4 and 5, patients in group B were further divided into those with fractures: i) extending more than halfway into the medial cortex $(\mathrm{n}=68)$, ii) extending less than halfway into the medial cortex $(n=22)$, and iii) experiencing complete extension
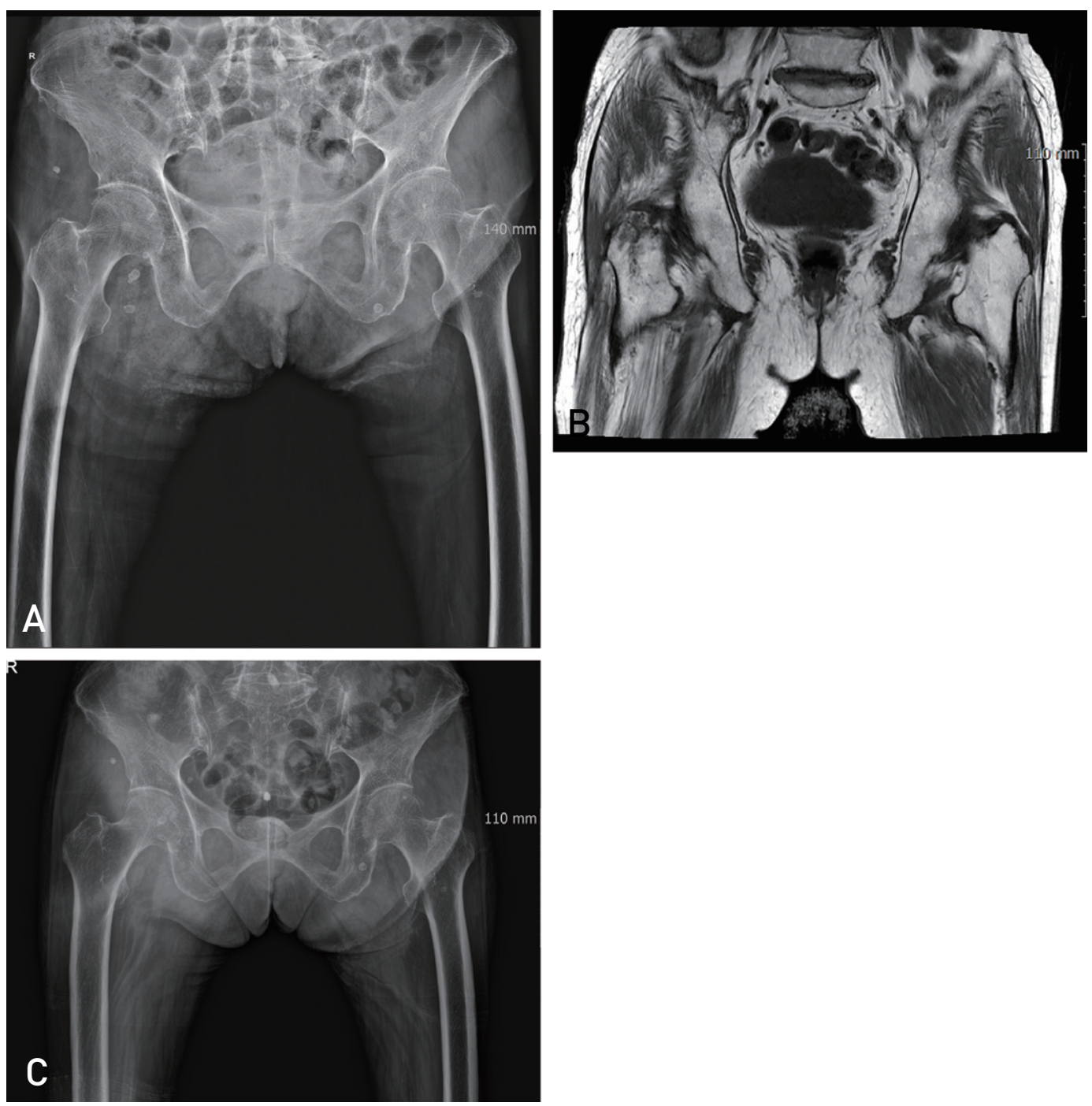

Fig. 4. An 89-year-old woman visited the hospital with right hip pain caused by a fall from height. (A) Plain radiography indicated an isolated greater trochanteric fracture. (B) Magnetic resonance imagingscans further revealed an occult intertrochanteric fracture; however, the fracture was limited to less than half of the intertrochanteric area. (C) Conservative treatment was performed. 


\section{Hip \& Pelvis}

Hip Pelvis 31(1): 23-32, 2019

across the ITC region $(n=16)$.

We used Feldman's fracture system ${ }^{5}$ to classify the 83 patients who underwent MRI in group B; 41 (49.4\%) were classified as type I (more than halfway to medial cortex), 24 (28.9\%) were classified as type II (type I plus extension of fracture to diametaphysis), 15 (18.1\%) were classified as type III (less than halfway to the medial cortex), and 3 (3.6\%) were classified as type IV (type I plus superior extension of fracture to the base of the femoral neck) (Fig. 6).

The average ages were $66.30 \pm 14.36$ years (range, 36 81 years) and $73.57 \pm 13.16$ years (range, $34-94$ years) for groups $\mathrm{A}$ and $\mathrm{B}$, respectively; there was no significant difference in age between the two groups $(P=0.104)$. The mean BMD had T-scores of $2.27 \pm 0.8$ (range, -1 to -3.4 ) and $-3.08 \pm 1.2$ (range, -0.3 to -5.4 ) in groups $\mathrm{A}$ and $\mathrm{B}$, respectively; this difference was significant $(P=0.087)$. There was no significant difference between the two groups relating to the distribution of gender, accident mechanism, and plain radiographic findings $(P=0.736, P=0.870, P=1.000)$ (Table 1).

Conservative treatment was performed in all 10 cases in group A and 28 cases in group B. The remaining 62 patients in group B underwent surgical treatment. In group B, 22 of the 28 cases had incomplete fractures less than halfway to the medial cortex, four patients were denied surgery, and two had general conditions that were too poor to conduct surgery. The remaining 62 patients with occult ITC fractures had damage that exceeded half of the diameter of the metadiaphyseal region and received surgical treatment using a two-hole dynamic hip screw without reduction. There were no complications related to surgery.
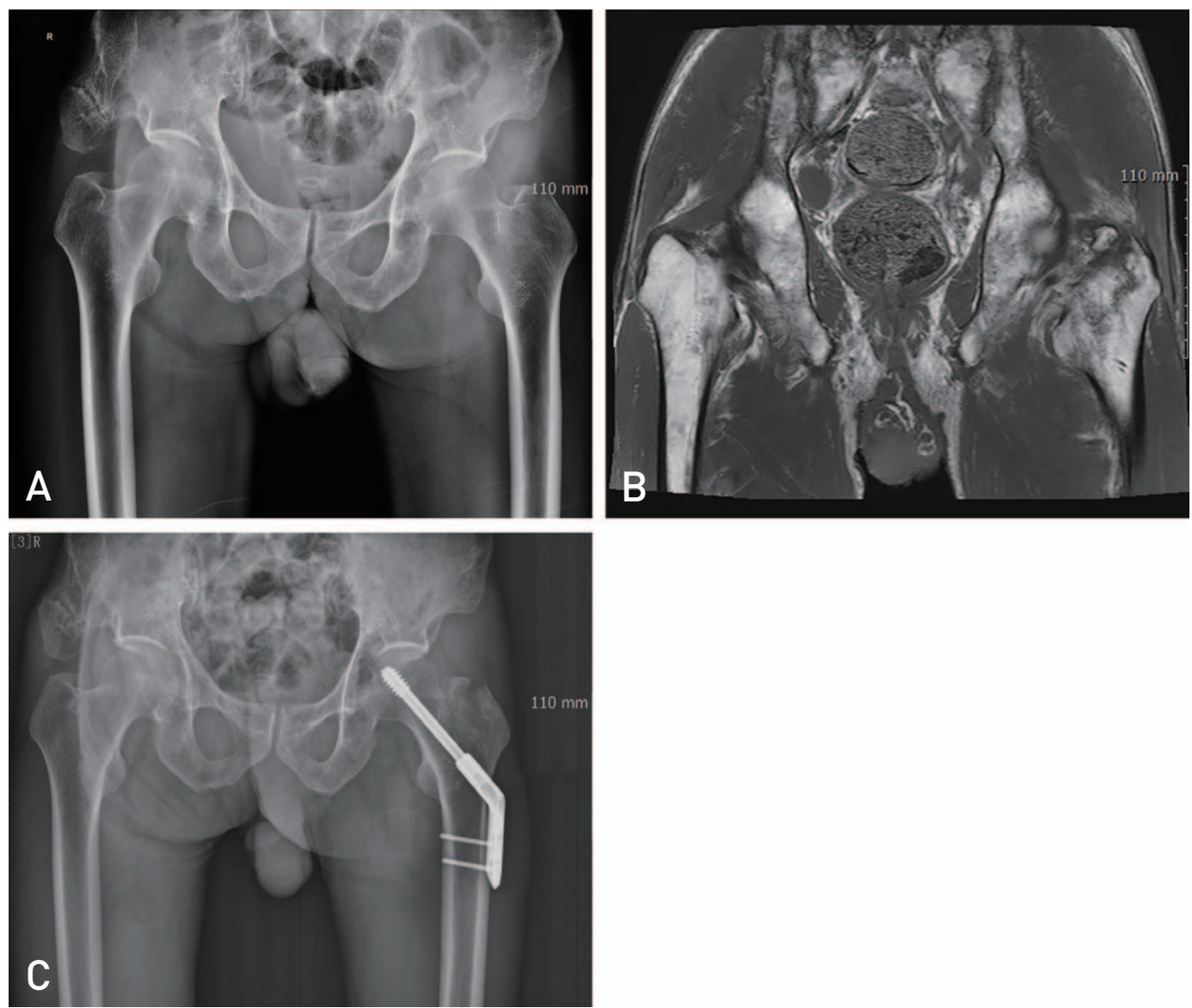

Fig. 5. A 75-year-old male visited the hospital with left hip pain caused by a fall from height. (A) Plain radiography indicated an isolated greater trochanteric fracture. (B) Magnetic resonance imaging scans further revealed an occult intertrochanteric fracture that was more than half of the intertrochanteric area. (C) Surgical treatment was performed using two-hole dynamic hip screws. 


\section{Hip \& Pelvis}

Jongho Noh et al. Isolated Greater Trochanteric Fracture with Occult Intertrochanteric Fracture

\section{DISCUSSION}

When hip trauma occurs, occult fractures-which are generally not identifiable using plain radiography-are assumed to be present in $2 \%$ to $10 \%$ of all pelvic and hip joint areas; these occult fractures can include femoral neck, ITC, and pelvic pubic rami fractures ${ }^{6}$. However, recent studies have reported occult fracture rates of $35 \%$ to $39 \%$ owing, at least in part, to advances in imaging equipment technology $y^{7,8}$. Also, if plain radiography indicates an isolated greater trochanteric fracture, in many cases further evaluation reveals that the fracture is extended to the ITC area. Previous studies have shown these extended fractures occur in between $28.5 \%$ and $94.5 \%$ of patients; however, these studies

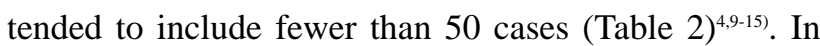
this study, only 10 of 100 cases wherein plain radiography indicated isolated greater trochanteric fractures were confirmed to be isolated greater trochanteric fractures alone; the remaining 90 cases were found to be occult ITC when further analysis with advanced imaging techniques was employed. To our knowledge, this is the largest ever study evaluating this phenomenon and suggests that additional imaging modalities may be necessary to better diagnose the extent of the patient's injury if plain radiography reveals an isolated greater trochanteric fracture.

In group B, there were only 15 cases (18.1\%) of Feldman type III fractures, which required only conservative treatment for an occult ITC. However, the remaining cases $(81.9 \%)$ were of fracture types that generally required surgical treatment. Type IV was present in 3 cases (3.6\%), suggesting that not only occult ITC, but also occult femoral neck fractures were seen, thus emphasizing the importance
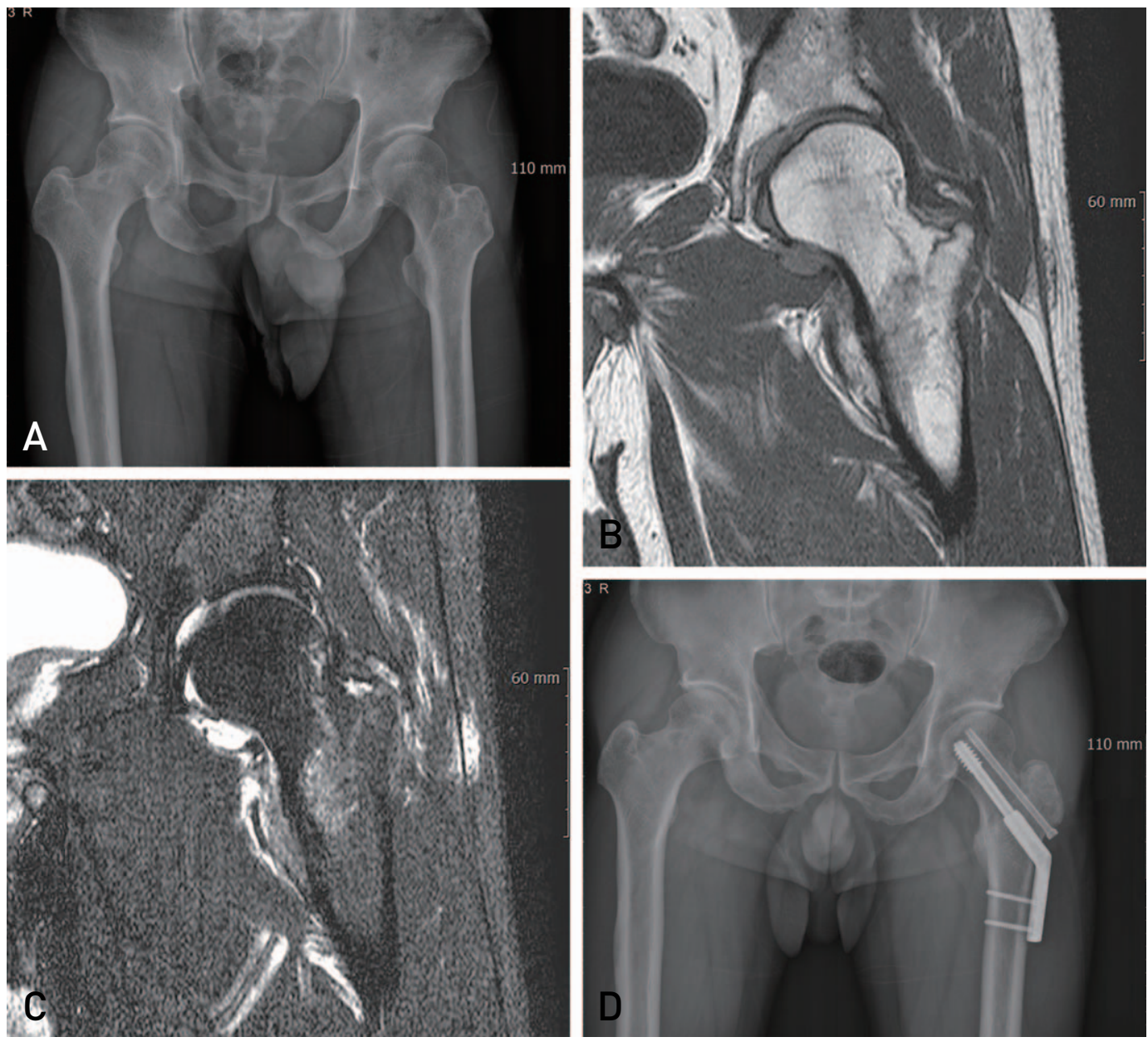

Fig. 6. A 41-year-old male visited the hospital with left hip pain caused by a fall from height. (A) Plain radiography indicated an isolated greater trochanteric fracture. (B, C) Magnetic resonance imaging scans further revealed an occult intertrochanteric fracture extended to more than half of the intertrochanteric area and the base of the neck. (D) Surgical treatment was performed using two-hole dynamic hip screws. 
of utilizing MRI.

There was also no significant difference between the two groups in terms of sex, age, accident mechanism, direction, BMD, and plain radiographic findings. This suggests that when an isolated greater trochanteric fracture is identified on plain radiography, additional tests to confirm the presence and precise anatomical involvement of an occult ITC fracture should be conducted, as such fractures may occur even in the absence of particular risk factors.

When plain radiography indicates that a patient has an isolated greater trochanteric fracture, additional evaluations by MRI, CT, or bone scanning can be performed. Of these, there is no doubt that MRI is most sensitive in diagnosing occult ITC fractures ${ }^{7,8,16}$. In this study, 4 out of 39 cases of bone scans did not match, and all of them appeared to have isolated greater trochanteric fractures in the bone scans that were actually occult ITC fractures on the MRI scans. Thus, bone scans can be estimated to be $90 \%$ sensitive, which is lower than the previous known sensitivity of $93 \%$ to $98 \%{ }^{177}$. Mettler and Guiberteau ${ }^{18)}$ reported that bone scans had an $80 \%$ sensitivity on accident day, a $95 \%$ sensitivity 3 days after the accident, and a $98 \%$ sensitivity 1 -week after the accident. Because of these characteristics, a bone scan took 5.46 days to conduct, which was longer than the 2.65 days taken to conduct an MRI examination. Therefore, we were able to verify once again that MRI could be used to make a more accurate and a quicker diagnosis.

Both CT and MRI were performed in only 7 cases. So the reliability of the direct comparison between $\mathrm{CT}$ and MRI is limited in this study.

Deleanu et al. ${ }^{19)}$ reported that the initial diagnosis was obtained in $34.2 \%$ of patients at their first visit to the emergency room due to trauma to the hip, while $65.8 \%$ of patients were diagnosed by additional CT or MRI after 72 hours. In the case of CT, Haubro et al. ${ }^{20}$ reported that the degree of variance in the diagnosis was 0.47 to 0.87 depending on proficiency in interpretation. In a study by Yun et al. ${ }^{21}$, the initial cost of imaging was higher than that of MRI. However, MRI was found to be the most cost-effective in terms of diagnosis/misdiagnosis rates, and treatment cost.

In addition, single-photon emission CT, dual energy $\mathrm{CT}$, or ultrasonography were attempted for the evaluation of occult ITC fractures. However, when compared to those of MRI or CT, their sensitivity and specificity were still very low, making it difficult to replace MRI or CT with these alternative methods. However, in special cases

Table 1. Baseline Characteristics of the Participants

\begin{tabular}{|c|c|c|c|}
\hline Characteristic & Group A $(n=10)$ & Group B $(n=90)$ & $P$-value \\
\hline Age (yr) & $66.30 \pm 14.36(36-81)$ & $73.57 \pm 13.16(34-94)$ & 0.104 \\
\hline Sex, male:female & $3: 7$ & $36: 54$ & 0.736 \\
\hline Accident mechanism & & & 0.870 \\
\hline Slip down & 8 & 82 & \\
\hline Fall down & 0 & 6 & \\
\hline Traffic accident & 2 & 2 & \\
\hline Fracture site, right:left & $4: 6$ & $43: 47$ & 0.746 \\
\hline Bone mineral density (T-score) & $-2.27 \pm 0.8(-1$ to -3.4$)$ & $-3.08 \pm 1.2(-0.3$ to -5.4$)$ & 0.087 \\
\hline Plain radiograph & & & 1.000 \\
\hline Transverse & 5 & 41 & \\
\hline Oblique & 4 & 36 & \\
\hline Comminution & 1 & 13 & \\
\hline Further study & & & $<0.001$ \\
\hline Isolated GT & 10 & & \\
\hline ITC, $<50 \%$ & & 22 & \\
\hline $\mathrm{ITC},>50 \%$ & & 68 & \\
\hline Comminution & & 16 & \\
\hline Treatment & & & $<0.001$ \\
\hline Conservative care & 10 & 28 & \\
\hline Surgery & 0 & 62 & \\
\hline
\end{tabular}

Values are presented as mean \pm standard deviation (range) or number only.

Group A: isolated greater trochanteric (GT) fractures group, Group B: occult intertrochanteric (ITC) fractures. 
Hip \& Pelvis

Jongho Noh et al. Isolated Greater Trochanteric Fracture with Occult Intertrochanteric Fracture

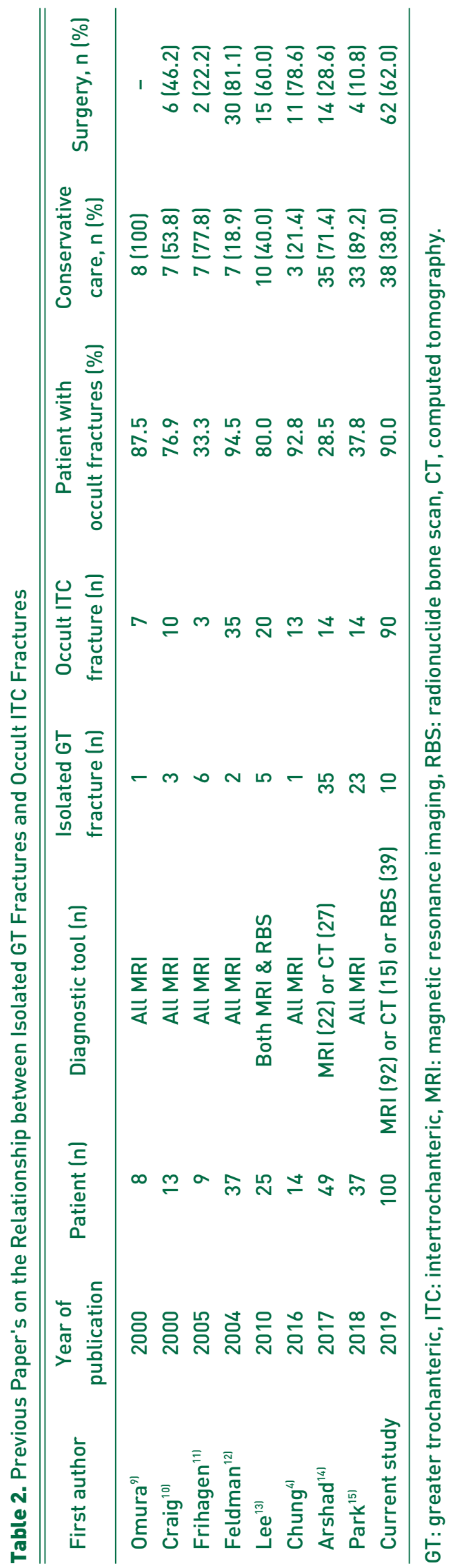

wherein MRI is not possible because of the presence of a pacemaker, etc., other methods may be tried ${ }^{22-25}$.

No definite treatment for occult ITC fractures has been established. However, it has been suggested that even when conservative treatments are performed, the success rate of these treatments is high, and that surgical treatment is essential if certain criteria are met.

A study by Omura et al. ${ }^{9)}$ showed that even if the fracture is extended to the whole ITC area, conservative treatment (i.e., bed rest for three weeks can be attempted). Alam et al. ${ }^{26)}$ also reported that conservative treatment alone could achieve bone union. Rubin et al. ${ }^{27)}$ proposed that bed rest along with early ambulation was also a treatment option. LaLonde et al. ${ }^{28)}$ have stated that if a fracture is limited to less than half the ITC area on the MRI, the patient could walk immediately using a walker.

On the other hand, Feldman et al. ${ }^{5)}$ and Feldman and Staron ${ }^{12)}$ encourage surgical treatment and performed surgery even when an isolated greater trochanteric fracture occurred because the hip motion and weight bearing extended from the greater trochanteric fracture to the ITC fracture. According to Craig et al. ${ }^{10)}$ and Schulz et al. ${ }^{29}$, surgical treatment is required when the fracture line on the MRI exceeds half of the ITC area. In response, Frihagen et al. ${ }^{11)}$ explained that if the occult fracture line is more than half of the ITC area, it increases the force on the inner layer of the cortical bone and increases the potential for complete fractures. Recently, Park et al. ${ }^{15)}$ has stated that the medial part is more important than the lateral part, and if a fracture occurs within one-third of the medial part, the potential risk is increased.

A limitation of this research is the retrospective nature of the study design. In particular, there may be risk factors other than those listed above, but they were not considered because of the lack of data. The study was also conducted by a single hospital, and surgical treatment was performed by one surgeon, so there may be limitations in extrapolating more broadly from this unique scenario and potential lack of diversity. This study focused on the frequency of occult ITC fractures in patients with preexisting radiographic evidence of isolated greater trochanteric fractures. Therefore, the outcomes of treatment were not taken into account. These factors should be the subject of a subsequent study. Nevertheless, this study covered 100 cases, which is meaningful in that it has analyzed a larger number of cases than previous studies. 


\section{CONCLUSION}

In this study, greater than $90 \%$ of isolated greater trochanteric fractures identified using plain radiography were reclassified as occult ITC fractures when analyzed with other imaging modalities. This finding strongly suggests that additional analyses should be conducted to test for the potential presence and precise anatomical involvement of an occult ITC fracture.

\section{CONFLICT OF INTEREST}

The authors declare that there is no potential conflict of interest relevant to this article.

\section{REFERENCES}

1. Milch H. Avulsion fracture of the great trochanter. Arch Surg. 1939;38:334-50.

2. Beloosesky Y, Hershkovitz A, Guz A, Golan H, Salai M, Weiss A. Clinical characteristics and long-term mortality of occult hip fracture elderly patients. Injury. 2010;41: 343-7.

3. Kim KC, Ha YC, Kim TY, Choi JA, Koo KH. Initially missed occult fractures of the proximal femur in elderly patients: implications for need of operation and their morbidity. Arch Orthop Trauma Surg. 2010;130:915-20.

4. Chung PH, Kang S, Kim JP, et al. Occult intertrochanteric fracture mimicking the fracture of greater trochanter. Hip Pelvis. 2016;28:112-9.

5. Feldman F, Staron R, Zwass A, Rubin S, Haramati N. MR imaging: its role in detecting occult fractures. Skeletal Radiol. 1994;23:439-44.

6. Rizzo PF, Gould ES, Lyden JP, Asnis SE. Diagnosis of occult fractures about the hip. Magnetic resonance imaging compared with bone-scanning. J Bone Joint Surg Am. 1993;75:395-401.

7. Chatha H, Ullah S, Cheema Z. Review article: Magnetic resonance imaging and computed tomography in the diagnosis of occult proximal femur fractures. J Orthop Surg (Hong Kong). 2011;19:99-103.

8. Rehman H, Clement RG, Perks F, White TO. Imaging of occult hip fractures: CT or MRI? Injury. 2016;47:1297-301.

9. Omura T, Takahashi M, Koide Y, et al. Evaluation of isolated fractures of the greater trochanter with magnetic resonance imaging. Arch Orthop Trauma Surg. 2000;120:195-7.

10. Craig JG, Moed BR, Eyler WR, van Holsbeeck M. Fractures of the greater trochanter: intertrochanteric extension shown by MR imaging. Skeletal Radiol. 2000; 29:572-6.

11. Frihagen F, Nordsletten L, Tariq R, Madsen JE. MRI diagnosis of occult hip fractures. Acta Orthop. 2005; 76:524-30.

12. Feldman F, Staron RB. MRI of seemingly isolated greater trochanteric fractures. AJR Am J Roentgenol. 2004;183: 323-9.

13. Lee KH, Kim HM, Kim YS, et al. Isolated fractures of the greater trochanter with occult intertrochanteric extension. Arch Orthop Trauma Surg. 2010;130:1275-80.

14. Arshad R, Riaz O, Aqil A, Bhuskute N, Ankarath S. Predicting intertrochanteric extension of greater trochanter fractures of the hip on plain radiographs. Injury. 2017;48:692-4.

15. Park JH, Shon HC, Chang JS, et al. How can MRI change the treatment strategy in apparently isolated greater trochanteric fracture? Injury. 2018;49:824-8.

16. Iwata $\mathrm{T}$, Nozawa $\mathrm{S}$, Dohjima $\mathrm{T}$, et al. The value of $\mathrm{Tl}$ weighted coronal MRI scans in diagnosing occult fracture of the hip. J Bone Joint Surg Br. 2012;94:969-73.

17. Holder LE, Schwarz C, Wernicke PG, Michael RH. Radionuclide bone imaging in the early detection of fractures of the proximal femur (hip): multifactorial analysis. Radiology. 1990;174:509-15.

18. Mettler FA, Guiberteau MJ. Essentials of nuclear medicine imaging. 5th ed. Philadelphia: W.B. Saunders Company; 2006, 269-70.

19. Deleanu B, Prejbeanu R, Tsiridis E, et al. Occult fractures of the proximal femur: imaging diagnosis and management of 82 cases in a regional trauma center. World J Emerg Surg. 2015;10:55.

20. Haubro M, Stougaard C, Torfing T, Overgaard S. Sensitivity and specificity of CT- and MRI-scanning in evaluation of occult fracture of the proximal femur. Injury. 2015;46:1557-61.

21. Yun BJ, Myriam Hunink MG, Prabhakar AM, et al. Diagnostic imaging strategies for occult hip fractures: a decision and cost-effectiveness analysis. Acad Emerg Med. 2016;23:1161-9.

22. Motomura G, Yamamoto T, Karasuyama K, Iwamoto Y. Bone SPECT/CT of femoral head subchondral insufficiency fracture. Clin Nucl Med. 2015;40:752-4.

23. Rieger B, Friederich NF, Rasch H, Hirschmann MT. [Nondislocated osteoporotic insufficiency fracture of the medial femoral neck. SPECT/CT makes the diagnostic difference]. Unfallchirurg. 2014;117:369-73. German.

24. Reddy T, McLaughlin PD, Mallinson PI, et al. Detection of occult, undisplaced hip fractures with a dual-energy CT algorithm targeted to detection of bone marrow edema. Emerg Radiol. 2015;22:25-9.

25. Safran O, Goldman V, Applbaum Y, et al. Posttraumatic painful hip: sonography as a screening test for occult hip fractures. J Ultrasound Med. 2009;28:1447-52.

26. Alam A, Willett K, Ostlere S. The MRI diagnosis and management of incomplete intertrochanteric fractures of the femur. J Bone Joint Surg Br. 2005;87:1253-5.

27. Rubin G, Malka I, Rozen N. Should we operate on occult hip fractures? Isr Med Assoc J. 2010;12:316-7.

28. LaLonde B, Fenton P, Campbell A, Wilson P, Yen D. Immediate weight-bearing in suspected isolated greater trochanter fractures as delineated on MRI. Iowa Orthop J. 2010;30:201-4.

29. Schultz E, Miller TT, Boruchov SD, Schmell EB, Toledano B. Incomplete intertrochanteric fractures: imaging features and clinical management. Radiology. 1999;211:237-40. 